\title{
EZ: A TOOL FOR AUTOMATIC REDSHIFT MEASUREMENT
}

\author{
B.Garilli, M.Fumana, P.Franzetti, L.Paioro, M.Scodeggio \\ IASF-Milano, INAF, Via Bassini 15, I-20133, Milano, Italy \\ O. Le Fèvre \\ Laboratoire d'Astrophysique de Marseille, UMR 6110 CNRS-Université de Provence, 38 rue Frederic \\ Joliot-Curie, F-13388 Marseille Cedex 13, France \\ S.Paltani \\ ISDC, Geneva Observatory, University of Geneva, ch. d' Ècogia 16, CH-1290 Versoix, Switzerland \\ R.Scaramella \\ INAF, Osservatorio Astronomico di Roma, via Frascati 33, 0040 Monte Porzio Catone (RM), Italy
}

\begin{abstract}
We present EZ (Easy redshift), a tool we have developed within the VVDS project to help in redshift measurement from otpical spectra. EZ has been designed with large spectroscopic surveys in mind, and in its development particular care has been given to the reliability of the results obtained in an automatic and unsupervised mode. Nevertheless, the possibility of running it interactively has been preserved, and a graphical user interface for results inspection has been designed. EZ has been successfully used within the VVDS project, as well as the zCosmos one. In this paper we describe its architecture and the algorithms used, and evaluate its performances both on simulated and real data. EZ is an open source program, freely downloadable from http://cosmos.iasf-milano.inaf.it/pandora.
\end{abstract}

Subject headings: Data Analysis and Techniques, Galaxies, Astronomical Techniques

\section{Introduction}

Thanks to larger telescopes and more powerful instruments, during the last decade we have witnessed an explosion in the size of spectroscopic surveys both in the nearby and in the more distant Universe: from the hundreds of objects of the surveys carried out in the early nineties (ESP, Vettolani et al. (1997), CFRS, Le Fevre et al. (1995)) to the thousands of galaxies of present days deep spectroscopic programs (zCosmos, Lilly et al. (2007), VVDS, Le Fèvre et al. (2005); Garilli et al. (2008), DEEP2, Coil et al. (2004)) and to the hundred thousands of more nearby surveys (e.g. 2dF, Colless et al. (2001), SDSS, Abazajian et al. (2009), 6dF, Jones et al.
(2009)) or to the hundred thousand or million objects of the just started programs (VIPERS, Guzzo (2009), WiggleZ, Drinkwater et al. (2009), BOSS, Schlegel et al. (2007)) and the few millions of galaxies of the future (e.g. EUCLID, Cimatti et al. (2009)).

Such an evolution in survey size requires an analogous step forward in the way the data are reduced and analyzed, making compulsory the use of reliable automatic tools. Among the various tasks to be done when carrying out a redshift survey, redshift measurement is one of the most demanding in terms of human resources and skills required. Historically, the rusao (Kurtz \& Mink 1998) package within IRAF has been the first widely used 
program which has been adopted for redshift measurement. It makes use of emission lines (emsao task) and of the continuum shape (xcsao task), on the basis of a correlation of the spectral continuum against galaxy or stellar templates, implementing the algorithm originally proposed by Tonry \& Davis (1979). Although very powerful, it does not foresee to weigh spectral features by an error spectrum. When the VIMOS-VLT Deep Survey (from here on, VVDS) started, it was immediately understood that this could be a major drawback in using rvsao within that project: VIMOS Low Resolution Red (LR-Red) spectra, like those obtained within the VVDS project, are affected by heavy fringing redwards of $\sim 8000 \AA$. As a consequence, spectra show features which are purely due to fringing but which can easily be mistaken with emission lines by any automatism, as well as by humans, unless they are properly weighted. Furthermore, if both emission and absorption lines are to be used, some scripting is required to couple together results from the pure cross correlation algorithm and the emission lines search one. Last, but not least, the IRAF implementation of rvsao did not allow a direct interface with the VIPGI package used for VVDS Data reduction (Scodeggio et al. 2005).

For all these reasons, we have decided to develop EZ (Easy redshift, pronounced "easy"), a new tool which inherits from the rvsao experience and the cross correlation technique, but allows to be easily plugged within VIPGI and could also be used as a VO-compliant tool via SSAP (Tody et al. 2008) or other communication protocols. From the user's point of view, the most important requirement was to be able to run the program both in a completely unsupervised and in fully interactive mode, with a user friendly graphical interface to inspect the results obtained using different parameters or templates. Furthermore, it must be possible to use templates which do not necessarily cover the full spectral range for any redshift value. Finally, it has to be easily usable for different projects, i.e. all parameters must be defined either through a project dependent parameter file or via command line. EZ has been initially based upon the prototypes KBred (Scaramella 1999, unpublished) and YAZ (Scaramella 2004, VVDS team Internal report), with which the first 10000 VVDS spectra were measured, and which contained the correla- tion and fitting algorithm described below. In section 2 we illustrate the rationale of EZ. Section 3 gives a short illustration of the software architecture and the most important algorithms used are illustrated in section [4. Section [5] describes in full how a redshift can be measured by EZ and section [6 illustrates how a reliability flag is attached to the best solution proposed. In sections 7 and 8 we evaluate the performances on simulated and real data respectively. Finally section 9 shows how much time and efforts can be spared using a tool like EZ when carrying out a large redshift survey.

\section{EZ basic concepts}

There can be several approaches to perform redshift measurement: from the most simple ones (like emission line finding or cross correlation) to more sophisticated ones, like bayesian approach or principal component analysis. Within EZ, we have chosen to develop a kind of expert system: the core of EZ is a decisional tree which tries to mimick the decisional pattern followed by astronomers when measuring redshifts from spectra. When an astronomer manually measures a redshift, his brain almost unnoticeably performs several functions: he looks for the existence of emission lines, and if there are some, looks whether they match on a single redshift solution; he looks at the shape of the continuum, to find out whether the object is an early type galaxy, and in that case searches for a D4000 break and $\mathrm{Ca} \mathrm{H}$ and $\mathrm{K}$ absorption lines; discards parts of the spectrum affected by heavy noise and spurious features, like zero orders or sky subtraction residuals; possibly, looks at the raw 2dimensional spectrum to decide whether emission features are real lines, or sky subtraction residuals. The basic idea we have tried to implement in EZ is to allow the user to combine the available functions in the most appropriate way for the data at hand, thus building new user defined functions and methods. At the upmost level, a redshift measurement decisional tree can be built, which mimicks the decisional path followed by an astronomer to get to the measure of the redshift. Such decisional path can differ from data set to data set, and for this reason several decisional trees can coexist in EZ, exactly as several implementations (methods) of the same function can coexist. 
EZ implements several functions to perform the different tasks required: read data files and templates, subtract the continuum from a spectrum, find emission lines, measure the redshift, measure spectral features. Each of these functions, in turn, supports different methods, i.e. can use different algorithms to carry out that particular task: for example, fitting can use a chi-square minimization or some more sophisticated algorithm, correlation can be performed weighting data by a noise spectrum or without any weight, data can be read from fits or ascii files.

Before coming to a detailed description, it is worth mentioning the importance that spectral templates have during the redshift measurement process. Whenever the solution relies on correlation or fitting methods (i.e. is not based solely on emission lines matching), the reference templates used in the process should reproduce as much as possible the spectra to be analyzed. It is not always straightforward to have at hands templates for all possible galaxy types and covering a wavelength range as large as to cover from the UV to the NIR rest frame. Nor the same template library is necessarily the best suited for all projects. EZ does not provide templates itself, but allows the user to have different template suites and use those he thinks are more suitable for that particular data set. Furthermore, it is possible to specify a minimum range of overlap between the template and the spectrum to be used. This feature eases a bit the problem of providing templates covering an extremely large wavelength range when dealing with data sets sampling galaxies from the nearby universe up to $z>4$.

\section{Software architecture}

EZ is implemented in Python in the form of a command-line interpreter. It consists of a main Python class which imports other classes dedicated to redshift estimation, service functionalities, file I/O, line finding, line matching, and others. A complete description of the EZ classes is provided in the downloadable user's manual. In general, only the higher level methods are used (e.g. the decisional tree) from within the EZ environment, but experienced users can directly use the Python shell to import the various classes and combine lower level methods in different ways, thus exploiting at full the flexibility of the package. Even at the higher level, several sessions (i.e. instances of the same classes) can coexist, and users can directly compare the results obtained using different methods or functions.

Algorithms are implemented in Python, or in C for the most CPU intensive tasks: all algorithms implemented in $\mathrm{C}$ are called through a unique $\mathrm{C}$ interface, so that all of them can easily be used from a Python environment. All the classes are built in such a way that they can be imported as modules from any other Python code as well as from the Python shell. The main class contains methods that return the best solution obtained as well as a "theoretical" spectrum for a given template and a given redshift normalized to the input spectrum. Any other Python code can simply import the main class and then directly handle the proposed solution according to needs.

While EZ is primarily developed as a commandline tool, a gtk based graphical interface is available to the user. By design, it is "merely" an interface to the command-line interpreter: it shows the spectrum and the associated noise spectrum, allows superposition of the best fitting solution, lists the other (less probable) solutions and allows to overplot them onto the spectrum. An example of the Graphical User Interface is shown in Fig. 11. In this figure we show a low signal to noise spectrum with the purpose of demonstrating EZ capabilities also on low quality data.

\section{Algorithms}

\subsection{Emission line finding}

One way to measure a redshift is to look for emission lines on to which to anchor a solution. The basic concept of an emission line search algorithm is to look for sharp peaks in the spectrum, as candidate emission lines, and then see whether such peaks can be matched with a single redshift solution. In presence of fringe patterns which highly resemble emission lines, the main problem is to discard those peaks which are very likely fake features, without loosing true lines. The implemented procedure first builds a rough peak list containing the position of all pixels showing a flux 
above a user defined significance threshold. A gaussian is then fitted to each of such positions, and only those peaks for which the width of the fitting gaussian is within some user defined limits are retained: the minimum and maximum width of a line depends on the resolution of the spectrum, and as such are configurable. This check discards peaks due to non or badly cleaned cosmic rays, which are too narrow to be a real line. Among the remaining line candidates, a further check is made whether the real peak flux is within a factor of two from the fitted gaussian peak. This allows to discard most of the fake peaks due to fringing which often have an irregular shape. Depending on spectral resolution, partially resolved lines can also be poorly represented by a gaussian. For this reason such check can be tailored or altogether suppressed through user parameters. Finally, the significance of the peaks is computed, subtracting a local continuum and computing the ratio between the peak height and the noise weighted local continuum: only peaks showing a significance above a pre defined $\sigma$ (usually 4 or 5 ) are retained. As the noise is stronger in fringing affected regions, spurious peaks with a gaussian shape get discarded by this check. The lines thus identified are further subdivided into strong and normal lines, according to whether the peak value is higher than strongcut $* \sigma$ where strongcut is a user defined parameter. Strong lines are treated differently within the decisional tree (see section 5).

Finally, the algorithm tries to match all or some of such peaks to known combinations of emission lines at different redshifts. The lines to be used for the matching are defined in a configuration file, and can be changed at wish, according to the redshift range or the type of object explored by the survey.

\subsection{Correlation}

Before applying the correlation both the spectrum and the template are continuum subtracted so that what drives the results are the local features (such as weak emission lines, absorption lines, spectral breaks). Each available spectral template is redshifted to a given redshift and the correlation function is computed. During the computation, each pixel can be optionally weighted by its associated noise. In the noise weighted approach, the correlation function has the form:

$$
c(z)=\frac{\sum_{j \in \Lambda} \frac{\left(s_{j}-\bar{s}\right)\left(t_{j}-\bar{t}\right)}{n_{j}}}{\sum_{j \in \Lambda} \frac{\sqrt{\sum_{j \in \Lambda}\left(s_{j}-\bar{s}\right)^{2}} \sqrt{\sum_{j \in \Lambda}\left(t_{j}-\bar{t}\right)^{2}}}{n_{j}}}, z \in \Theta
$$

where $s_{j}$ and $n_{j}$ are the spectrum flux and noise at pixel $j$ respectively; $\bar{s}$ is the spectrum mean computed as

$$
\bar{s}=\frac{\sum_{j \in \Lambda} s_{j} / n_{j}}{\sum_{j \in \Lambda} 1 / n_{j}}
$$

$t_{j}(z)$ is the interpolated flux of the template at pixel $j$, once put at redshift $\mathrm{z} ; \bar{t}$ is the template mean; $\Lambda$ is the wavelength range in use and $\Theta$ is the redshift range to explore. Each template does not necessarily cover the full spectral range, in other words for different templates and redshifts the correlation function can be computed using a different number of points. In order to compare the results obtained for different templates, the value of the correlation function is normalized to the number of points used $(N)$.

$$
c(z)_{\text {red }}=\frac{c(z)}{N}
$$

Once the correlation function is produced for a particular template, the highest peak is returned as the best correlation solution for that template.

\subsection{Fitting}

The fitting of a spectrum against a template is performed using a non-continuum subtracted spectrum, so that the results are affected also by the overall shape of the underlying continuum. It uses a standard least squared metric: each spectral template available is redshifted to a given redshift and the mean square deviation between spectrum and template is computed as

$$
\chi_{\text {red }}^{2}=\frac{\sum_{j \in \Lambda}\left(\frac{f_{j}-A t_{j}}{\sigma_{j}}\right)^{2}}{N}, z \in \Theta
$$

where $f_{j}$ and $\sigma_{j}$ are the spectrum flux and noise at pixel $j$ respectively; $t_{j}(z)$ is the interpolated flux of the template at pixel $j$, once put at redshift $z ; \Lambda$ is wavelength range in use and $\Theta$ is the redshift range to explore; $N$ is the number of 
data points used in the computation. Each template does not necessarily cover the full spectral range, in other words for different templates and redshifts the $\chi^{2}$ can be computed using a different number of points. In order to compare the results obtained for different templates, the $\chi^{2}$ is divided by the number of data points used The normalization constant $A$ is computed as:

$$
A(\Lambda, z)=\frac{\sum_{j \in \Lambda} \frac{f_{j}}{\sigma_{j}^{2}}}{\sum_{j \in \Lambda} \frac{t_{j}}{\sigma_{j}^{2}}} .
$$

The minimum reduced $\chi^{2}$ for each template and redshift range is returned.

\subsection{The solve method}

When no emission lines have been found in a spectrum, or when the lines found do not point to one single solution, redshift determination can be done using a correlation, or a fitting procedure, as described above. The solve function within EZ is meant to combine cross correlation, fitting or any other elementary method which can lead to a redshift solution. As all other functions, it can have different methods, i.e. the various elementary algorithms can be combined in different ways according to wishes.

The current implementation of the solve method foresees the use of cross correlation first, and a further fitting step. We have noticed that in the VVDS and zCosmos data sets, the highest peak of the correlation function as described above is not always the best solution, and this depends mainly on the fact that the noise spectrum does not (negatively) weigh enough the spurious features of the fringing. On the other hand, also the simple fitting does not give satisfactory results in terms of picking up the correct redshift. A sequential usage of the two methods, instead, has proven to be the one giving the best results for our data. First a correlation of the spectrum against each template is performed: as described above, this step allows to properly take into account the local features of the spectrum, like spectral breaks, absorption lines and weak emission features. At the end of the correlation, for each template the $n$ redshifts (where $n$ is a user defined parameter) corresponding to the highest correlation peaks are retained. At this point, we are faced with $n \times m$ solutions (where $m$ is the number of available templates). To discriminate among them, we use the fitting procedure: as fitting is performed using the spectrum without subtracting the continuum, the overall spectrum shape plays a role into getting a lower reduced $\chi^{2}$, and can help to pin point the correct redshift. Finally, the solution giving the minimum reduced $\chi^{2}$ is chosen.

\section{EZ decisional tree}

The core of EZ is the "decisional tree", which tries to mimick the human decisional process applied during redshift measurement. This is where one defines the actions to be performed, and in which sequence they must be carried out in order to measure a redshift. Each logical block of a decisional tree makes use of the lower level algorithms available. Several decisional trees can coexist in EZ: each one acts differently in different situations, and this can be useful to better tune the measurement process according to the kind of data at hand. For example, in a survey where stars have been a priori removed, it may be useful not to check for M stars, thus reducing the degrees of freedom of EZ, and consequently the possibility that it takes a false track. As an example, we illustrate here the decisional tree we have implemented for the VVDS and zCosmos surveys. The input consists of one or several observed spectra with a noise spectrum associated to each of them. Following fig 2, the steps performed are as follows

1. Check if the object is an M type star: the characteristical wavy shape of the spectra of this kind of stars can be recognized by looking if around the expected position a very large gaussian can be fitted, and if the spectrum is steadily becoming redder and redder with increasing wavelength.

2. If such check is positive, in order to assign a best-fitting template to the object a fit is made using only M-star templates, and the redshift is set to zero.

3. If the $M$ star check has failed, search for emission lines in the spectrum; if lines are found go to step 4, otherwise go to step 8

4. Search for a match between lines. If one or more matches with at least 2 lines have been found go to step 5. otherwise go to step 6. 
5. Fit each of the possible solutions given by the matches above with emission lines templates. Choose the solution giving the minimum reduced $\chi^{2}$.

6. Check if strong lines have been found, (see 4.1). If this is the case go to step 7] otherwise go to step 8 .

7. Using only the redshifts satisfying matches with the one strong line, and only the emission line templates, compute the redshift using the algorithm defined by the solve method 4.4

8. Using all selected templates and the whole redshift range indicated by the user, compute the redshift using the algorithm defined by the solve method (4.4)

\section{Redshift reliability}

Complete automation of the redshift measurement process can be tricky when spectra are noisy (as they always are at the faint limit of a survey) or in presence of artifacts such as fringing correction residuals, so that it is by no means guaranteed, a priori, that the best solution proposed by $\mathrm{EZ}$ is also a correct solution. For this reason we have added the computation of an integer reliability flag which summarizes the goodness of the solution proposed. Such a flag is computed mimicking the kind of logical reasoning applied by an astronomer when trying to evaluate if a redshift is reliable or not. The flow is as follows

- each template is described by a "reliability" file. An illustration of such file for an Elliptical galaxy and for a StarBurst galaxy, as used with the VVDS and zCosmos projects, is given in Table 1] in the first column, the features which are tested are given, together with their wavelength (column 2). Each feature has a weight associated (column 5), according to the prominence the feature usually has in standard spectra. For example, in elliptical galaxies the D4000 break is the most prominent feature, thus its weight is higher than all other lines. On the other hand, if the feature is not found, then the redshift becomes suspect, thus a negative weight is associated in this case (column 6).
Some features are commonly found together with other features (e.g., the [OIII] doublet, or the D4000 which usually comes with Ca $\mathrm{H}$ and $\mathrm{Ca} \mathrm{K}$ absorption lines). The third column lists such secondary features, if applicable. If these features are found together with the main line, then the weight of the main line is increased to the value listed in column 4. Note that what is important is not the absolute value of the weight itself, but its relative value with respect to the other expected fetaures. The reliability files should be created according to user's needs and to the templates used.

- the "best fitting" template is redshifted to the measured "best redshift", and a correlation around each expected spectral feature is performed, taking into account the noise spectrum. The correlation value must be above a user defined threshold for the feature being considered as "found". In this step, observational constraints are taken into account: e.g. if one of the expected lines falls outside the observed spectrum (or too close to the border) it is ignored.

- if the "best solution" is a star also the "color" is computed, as the difference between the mean value in the bluer and redder part of the spectrum, and used as a "feature" (blue color for earlier star types). If the star type is M, a dedicated algorithm searches for the characteristic "wavy" shape and uses them as features found (or not found).

- if a feature has been found, then its correlation value is weighted according to the weight given to that feature in the reliability file for that template (column 4 or 5 in Table 11). Features listed in the reliability file, but not found, are negatively weighted (column 6 in Table 11).

- the weighted correlation values are summed up, and normalized by the number of lines which have been found, giving a rate

- finally, the rate is converted into a flag, according to the ratio of found features with respect to expected features and to the rate itself: the higher the rate and the number 
of features found, the higher the flag. The conversion is made in such a way that EZ reliability flags resemble as much as possible the VVDS reliability flags as defined in Le Fèvre et al. (2005) in terms of confidence level.

Flags thus computed range from 0 (solution is not reliable) to 4 (highly reliable solution). In Table 2 we summarize the confidence level of the different flags as defined in the VVDS project, and the criteria used by EZ to assign each flag.

\section{Performances on simulated data sets}

For any given spectrum, EZ gives in output the best redshift and the best fitting template, while the output of the reliability process is the flag and the number of features found. When evaluating performances, we should make a distinction between the performances of the redshift measurement methods, i.e. the capability of finding the correct redshift, and the performances of the reliability method, i.e. the capability of assessing the goodness of the solution.

To perform such evaluations, we have carried out two different sets of tests: a first set, using simulated spectra, and a second set, making use of real spectra from the VVDS and the zCosmos survey.

\subsection{Simulated test set}

Using simulated spectra to evaluate performances of an algorithm has the advantage that the answer is known beforehand, and thus results can be evaluated with no error margin. On the other hand, even if simulations are carried out as carefully as possible, such set can only give an upper limit to the performances, as simulated spectra never take into account all possible noise sources and defects existing on real data.

The procedure we used to simulate spectra begins with an input catalog of objects: in this case we have used the Cosmos Mock Catalog (from here on CMC), described in Jouvel et al. (2009). In brief, CMC is a simulated catalog built directly from the observed COSMOS (Scoville et al. 2007) catalog of Capak et al. (2007) and the COSMOS photometric-redshift catalog (Ilbert et al. 2009). In CMC, a redshift and a spectrophotometric type are associated to each galaxy of the COSMOS cat- alog, using a model fitting procedure of the photometric data. The resulting catalog contains a mix of galaxy populations which by construction is representative of a real galaxy survey. Emission line fluxes are also computed and magnitudes in a number of filters are made available. Details on the simulation of observed spectra will be given elsewhere (Franzetti et al., in preparation). To summarize the procedure, a rest frame spectrum, as can be obtained from galaxy model libraries, has been associated to each galaxy type as provided in the catalog (elliptical, early spiral, late spiral and starburst galaxy). In this step, line broadening due to galaxy velocity dispersion has been neglected, as at the resolution of the VVDS and zCosmos data this it is irrelevant. The monodimensional incident spectrum has been obtained by redshifting the rest frame spectrum and normalizing it so as to give the object apparent magnitude in the chosen selection band, $I_{A B}$. The incident spectrum has then been degraded for the VIMOS efficiency curve, as can be obtained from the ESO Exposure Time Calculator. In the noise calculation, we have taken into account the Poissonian noise (from both sky and object), the flat fielding accuracy and the electronic noise (for a detailed explanation of the different contribution see e.g. Newberry (1991)). The sky spectrum used has also been derived from ESO VIMOS exposure time calculator. We have compared our simulated spectra with those which are obtained from ESO exposure time calculator, once the same exposure time, galaxy type and apparent magnitude are used, and the results are extremely similar both in terms of signal to noise as a function of wavelength, and in terms of sky subtracted spectrum. It is important to note that our simulated data set includes the electronic noise, the poissonian noise, the flat fielding accuracy but does not include the effect of fringing.

We have simulated $\sim 11000$ galaxy spectra, in the magnitude range $17.5 \leq I_{A B} \leq 22.5$ using the same exposure time as for the VVDS wide survey and the zCosmos bright survey (Garilli et al. (2008) and Lilly et al. (2007)), and the VIMOS LR red grism. CMC does not contain stars, but we are interested to check EZ performances also on different types of stars, as the selection function of galaxies for surveys never completely succeeds in excluding stars on the basis of photometry. Thus 
we have simulated 11000 stars, with magnitude ranging from 17.5 to 22.5 , of different spectral types. The Pickles stellar library has been used for their model spectra. On this simulated data set, we have run EZ in totally blind unsupervised mode and obtained for each object a redshift and a redshift flag. The template set we have used in $\mathrm{EZ}$ is the one obtained within the VVDS project and built from the VVDS data themselves: it comprises two templates for early type galaxies, one template for Sbc galaxies, one for Scd galaxies and 3 for starburst galaxies, with different intensities of emission lines and line ratios. These templates extend from $\sim 3500 \AA$ to about $\sim 8000 \AA$ rest frame and are particularly suited to search for a redshift solution within the redshift range 0 to 2.0, a range which well matches the redshift range of the simulated data given the magnitude cut we have imposed, in spectra covering the observed range of the VIMOS LR-Red grism. The measured redshift has then been compared to the real input value, and classified as correct when the measured value is within $10^{-3}$ of the real value, this limit corresponding to the theoretical redshift error given by the grism resolution.

\subsection{Global performances on simulated spectra}

In Table 3 the results of such comparison are summarized: for galaxies and stars separately, as well as for the whole sample(which has comparable numbers of galaxies and stars), we give the success rate (computed as the ratio between correctly retrieved redshifts and total number of objects), the number of correct redshifts, and the total number of objects to which EZ has assigned the given flag. Results for each object type are splitted per EZ redshift flag.

Table 3 shows that on simulated data, EZ is exceptionally good at retrieving the correct redshift, the success rate being $97 \%$ on the whole sample. In spite of the large number of simulated spectra, very few objects are classified with a reliability flag of 1 or 2 , so that the average success rate for these flags has not the same statistical significance as the other flags.

Going deeper in the analysis of results, we note that stars are practically always recognized as such, even if the flag associated to their measure- ment is extremely low in half the occurrences. This is intrinsic to the way we associate flags: for types younger than $\mathrm{K}$, stellar spectra are poor in spectral features in the wavelength range explored. At the resolution we used, only $\mathrm{NaD}$ and $\mathrm{H} \alpha$ absorption lines are clearly visible. Furthermore, $\mathrm{NaD}$ falls very near to a strong sky line, and it is often not detected due to the higher noise. Thus only one out of two features is clearly detectable. This explains the frequency of zero flag objects in this category. However, only 191 galaxies $(<2 \%)$ are erroneously mistaken for stars, while among the 10762 objects classified as galaxies, only 19 were actually stars.

\subsection{Dependence on magnitude and red- shift}

We have shown that on the simulated test set, the global success rate is very high (97\%). Still we expect it to show a trend with object magnitude, which can be considered a proxy for signal to noise ratio for a fixed exposure time. This is shown in figure 3. bottom panel, where we plot the success rate obtained as a function of magnitude. Black circles show the success rate obtained considering all flags above 1, while red crosses show the success rate obtained considering very secure redshifts (flags 3 or 4) only. Even using all flags above 1 (secure redshifts), it is evident that the fainter objects have an increasingly lower success rate, even if it stays always well above $80 \%$.

The top panel of figure 3 shows the success rate as a function of the true redshift of the object. The drop in success rate for $z>1.4$ for secure redshift flags is not very surprising: between $\mathrm{z}=1.4$ and $\mathrm{z}=1.5$, both the $[\mathrm{OII}]$ line and the D4000 break start to fall out of the observed wavelength range, and the redshift can be secured only for very few galaxies. In other words, we are entering in the redshift desert regime, were observations in the red visible part of the spectrum are known to be inefficient. Possibly more surprising, at first glance, can be the drop in success rate observed above $\mathrm{z}=0.9$ when only very secure redshifts are considered. This is mainly due to the way we compute the reliability of the redshift: at $\mathrm{z}=0.9,[O I I I]_{a}$ and $\mathrm{H} \beta$ lines progressively fall out of the observed wavelength range, so that the only strong and easily detectable line for late objects types remains 
[OII]. For this reason, many objects get a flag 9 (only one strong line). On the other hand, going higher in redshift objects become fainter, and their continuum gets noisier: therefore also early type objects are more difficult to measure and to get a very secure flag is even harder.

\section{Performances on real data sets}

We have tested EZ performances on the three data sets of the VVDS Deep survey (Le Fèvre et al. 2005), the VVDS Wide survey (Garilli et al. 2008) and the zCosmos survey (Lilly et al. 2007). The three data sets have different characteristics, and are complementary to evaluate EZ performances: the VVDS Deep sample is cut to a deeper apparent magnitude limit $\left(I_{A B} \leq 24.0\right)$, thus its redshift distribution extends beyond $\mathrm{z}=1.5$ with significant numbers. On the contrary, the VVDS Wide is cut at a brighter limit $\left(I_{A B} \leq 22.5\right)$, and has a stronger star contamination. The zCosmos sample, finally, has the same depth as the VVDS Wide sample, but it has been observed with a higher resolution grism and stars have been a priori discarded on the basis of their morphologies and spectral energy distributions: the criteria used were intentionally quite conservative and a small fraction of stars is expected in the sample (Lilly et al. 2007). All redshifts for these three samples have been manually measured by two different persons (also using beta versions of EZ in interactive way), discrepant measures have been reconciled, and a flag has been assigned to each redshift, corresponding to a confidence level as described in table 2 column 2, a long and time consuming procedure which has requested big efforts on the part of the people involved. A posteriori, we have run EZ in blind and unsupervised mode on the data sets, using only objects with a measured redshift between 0 and 2.0. By comparing the results found by EZ with those published by the VVDS and zCosmos consortia, we can evaluate EZ performances on real data. We expect such performances to be worse than what obtained on simulated data sets, as now all possible error sources are present, last but not least the fringing above $\sim 8000 \AA$.

Table 4 and Figure 4 show the success rate of the EZ blind measurement in the three different samples. As expected, the global performances are not as exceptional as for the simulated data, rang- ing from $\sim 68 \%$ to $\sim 76 \%$ in the best case of the zCosmos data. However, a straight comparison is not totally fair: as declared in the surveys themselves and reported in Table 2, measured redshifts are never claimed to be $100 \%$ correct. The last column of Table 4 reports the weighted success rate: for each EZ flag, the non concordant redshifts have been weighted in number according to the humanly given flag, so that discordant objects having a human flag 1 count by half, if they have human flag 2 or 9 they are weighted $75 \%$, if they have human flag 3 and 4 they are weighted $90 \%$ and $95 \%$ respectively. Using such weights, the success rate obviously increases, as the uncertainty of the humanly measured redshifts is properly taken into account.

Still, the success rate remains lower than what we had for simulated data, especially in the VVDS samples. The main reason for this are the fringing features, which if not properly weighted are easily mistaken for real features by any automatic procedure. As explained in the previous section, in the simulated data set we have not included the effect of fringing, and for this reason simulated spectra are much less noisy redwards of $8000 \AA$ and EZ is not fooled by spurious emission features. The noise spectrum associated to real data should help in weighting considerably less such features, but when fringing features are particularly strong, the noise spectrum, as currently computed, is not enough to completely neutralize their effect on the emission line finding algorithm. As a test for this hypothesis, we have run EZ on these same data sets without using the noise spectrum and the resulting success rate is considerably lower. In the zCosmos sample, thanks to the larger dithering width the fringing pattern is better removed from the data and the associated noise spectrum better allows to take into account any residual. This is one of the reasons why in the zCosmos sample the success rate for the most secure EZ flags is the same as for simulated data. A second important effect is the presence of the zero order in the extracted spectra: if this is not removed, it is mistaken for an emission line and the redshift is obviously wrong. Again, zCosmos data are less affected because the multiplexing of those observations is much lower than for the VVDS data. Finally, also the observational conditions have an impact on the data quality and the redshift mea- 
surement: clouds and bad seeing diminish the $\mathrm{S} / \mathrm{N}$ ratio, a lower exposure time as well as a too high airmass decrease the signal to noise, bad centering of the object in the slit increases slit losses. All these factors contribute to lower the global redshift measurement success rate with respect to the idealized case of simulations.

Looking deeper at the concordant measurements, it can be noted that the flag assigned by EZ is not always identical to that assigned by the astronomers. This is summarized in Table 5, where we compare EZ flag with the human flag for concordant measurements. In this table, we use only measurements obtained for galaxies, as we have already shown that when the object is a star the flagging system we have implemented gives low reliability values. We have grouped results for flags 2 and 9 , because experience has taught that astronomers' ideas on the two flags differ from person to person: when only one emission line is clearly visible, some people look at the continuum slope and assign a flag 2, others don't. Table 5 shows that the similarity of the two flagging systems is confined to the highest confidence bin: the vast majority of the most reliable measurements by EZ are considered very secure also by astronomers. Going to lower EZ flags, the astronomer's flag is usually higher than what judged by the automatic algorithm. This effect is not unexpected, since the EZ flagging system has been set up to be as reliable as possible, at the expenses of being too pessimistic in many cases. We must also consider that the criteria used by EZ and by an astronomer are not exactly the same: astronomers only grossly take into account the correlation with the continuum slope, while in absence of emission lines this is very important in an automatic tool. Also the lines to be searched must be very significant for EZ to take them into account, and the significance is weighted with the corresponding noise spectrum, while an eye measurement is more elastic in judgment, also because of the possibility of double-checking $1 \mathrm{D}$ with $2 \mathrm{D}$ spectra. Finally, the astronomer's judgment is subjective and changes not only from person to person, but also with the tiredness of the person performing measurements. An automatic evaluation, on the contrary, although more pessimistic, is always based on the same criteria.

As reported in the papers presenting the surveys
(Le Fèvre et al. (2005), Garilli et al. (2008) and Lilly et al. (2007)), a redshift has not been measured for all objects. These measurement failures are indicated in the catalogs with a conventional human flag of zero and no redshift indication, thus they are not included in table 4 Nevertheless, it is interesting to see which flag EZ gives to these critical data. In all the three samples we have considered, more than $80 \%$ of the spectra for which astronomers did not measure a redshift get a flag 0 or 1 from EZ, a clear indication of a highly unreliable solution. In the remaining few cases, EZ gets fooled by fake features, indicating that the noise spectrum is not accurate enough.

\section{Application on large extragalactic red- shift surveys}

The original purpose for which EZ has been developed is to ease and speed up the long and painful phase of measuring redshifts in large surveys, reducing human intervention as far as possible while keeping the redshift measurement success rate acceptable for the scientific purpose of the project. On the basis of the results shown in Table 3, and keeping in mind such purpose, we can explore which would be the limitations of adopting straightforwardly EZ results in measuring redshifts in a generic redshift survey performed in optimal conditions, as simulated data are, which explores the redshift range we have considered here, i.e. $0<z<2$.

We define as completeness the number of measured redshifts over the total number of spectra, while purity is the number of correctly recovered redshifts with respect to the measured ones. The results are given in table 6 accepting blindly EZ results for all objects with a measured redshift $>0$, irrespective of flag, the survey would be $>98 \%$ complete, with $<6 \%$ of wrong redshifts. According to the degree of completeness which can be sacrificed to purity, it is possible to retain only galaxies with very high reliability flag (3 or 4): as from table 6, in this case the resulting galaxy sample would be slightly less than $90 \%$ complete, but its purity would be extremely high (>95\%). If we apply these percentages to next generation surveys, where the number of spectra foreseen is of the order of few hundred thousands, the gain (meant as number of spectra for which human measurement 
can be avoided) in using a reliable automated redshift measurement tool is evident.

One may argue that real surveys are not always carried out in optimal conditions, real data are usually more difficult to treat than simulated data and as such the percentages given in table [6] are only upper limits. In section 8 , we have shown that EZ is extremely reliable to find the correct solution for spectra classified as very secure (the success rate being above $90 \%$ for EZ flag 3 and 4) even on real data, affected by different sources of noise. Using these encouraging results, we can extend the previous considerations to more realistic sets and estimate what would be the gain, in terms of number of spectra which can skip the human check, if we would fully trust the redshifts to which EZ assigns a flag 3 or 4 . This is summarized in table 7, where for each of the real samples we have analyzed we give the total number of objects observed, the number of objects for which EZ measured a highly reliable redshift, the purity of this highly reliable sample and the gain meant as the fraction of measurements which could be avoided. Such gain ranges from 40 to more than $50 \%$, and it translates into a factor of almost 2 sparing of human effort and time. Given the difficult nature of the data used for our tests (see the discussion on fringing effects in the previous section) such a number can be considered as a lower limit to the effective gain obtainable by using EZ in a large extragalactic redshift survey.

\section{Summary}

We have presented, EZ, an automated tool devoted to redshift measurement. The concept at the basis of the tool is the decisional tree, i.e. the sequence of operations to be performed to obtain a redshift. Such sequence of operations can be customized according to the kind of sample at hand: e.g. by discarding any check on stars, or using only emission lines without performing the correlations on the continua in the case of spectra obtained with an instrument operating in slitless mode.

EZ can be used both interactively, with the help of a graphical user interface, or totally blindly in unsupervised mode. It is developed in Python, with the bulk of computations performed in $\mathrm{C}$ to increase its computational speed. Its Python classes can be directly imported in any other Python based program, thus making it fully embeddable in any application.

Within EZ we have developed a method to assign a reliability flag on the measurement obtained, in order to mimic the kind of reasoning done by astronomers when assessing the goodness of the solution found. The implemented flagging system, though, is rather conservative.

We have tested EZ on VIMOS-like simulated data, and have shown that its performances are exceptionally good, the redshift measurement success rate being above $95 \%$.

We have blindly applied EZ to the VVDS-Deep, VVDS-Wide and zCosmos bright samples, and have demonstrated how this tool behaves very well also on real data. The success rate obtained is around $70 \%$, and rises above $90 \%$ for redshifts classified as very secure by astronomers.

Finally, we have shown that the adoption of a similar tool can save from a minimum of $50 \%$ to a maximum of $95 \%$ of the redshift measurement load, according to the quality of the data and to the degree of completeness and purity of results one is willing to sacrifice in favor of a fast output.

$\mathrm{EZ}$ is now being routinely and blindly run as part of the reduction process within the VIPERS survey, while a customized version has been set up to perform simulations of the E-NIS spectrograph (Cimatti et al. 2009).

EZ is an open source program, freely downloadable from http://cosmos.iasf-milano.inaf.it/pandora

We wish to thank the whole VVDS and zCosmos collaboration for their testing of the earlier versions of EZ and their helpful feedback. A special thank to Dario Maccagni, for his invaluable help in preparing the manuscript. This work has been fully supported by INAF, through funding by the Project Department via the Information Systems unit.

\section{REFERENCES}

Abazajian, K. N., Adelman-McCarthy, J. K., Agüeros, M. A., et al. 2009, ApJS, 182, 543

Capak, P., Aussel, H., Ajiki, M., et al. 2007, ApJS, 172,99

Cimatti, A., Robberto, M., Baugh, C., et al. 2009, Experimental Astronomy, 23, 39 
Coil, A. L., Davis, M., Madgwick, D. S., et al. 2004, ApJ, 609, 525

Colless, M., Dalton, G., Maddox, S., et al. 2001, MNRAS, 328, 1039

Drinkwater, M. J., Jurek, R. J., Blake, C., et al. 2009, ArXiv e-prints

Garilli, B., Le Fèvre, O., Guzzo, L., et al. 2008, A\&A, 486, 683

Guzzo, L. e. a. 2009, http://vipers.inaf.it

Ilbert, O., Capak, P., Salvato, M., et al. 2009, ApJ, 690,1236

Jones, D. H., Read, M. A., Saunders, W., et al. 2009, MNRAS, 399, 683

Jouvel, S., Kneib, J., Ilbert, O., et al. 2009, A\&A, 504,359

Kurtz, M. J. \& Mink, D. J. 1998, PASP, 110, 934

Le Fevre, O., Crampton, D., Lilly, S. J., Hammer, F., \& Tresse, L. 1995, ApJ, 455, 60

Le Fèvre, O., Vettolani, G., Garilli, B., et al. 2005, A\&A, 439, 845

Lilly, S. J., Le Fèvre, O., Renzini, A., et al. 2007, ApJS, 172, 70

Newberry, M. V. 1991, PASP, 103, 122

Schlegel, D. J., Blanton, M., Eisenstein, D., et al. 2007, in Bulletin of the American Astronomical Society, Vol. 38, Bulletin of the American Astronomical Society, 966-+

Scodeggio, M., Franzetti, P., Garilli, B., et al. 2005, PASP, 117, 1284

Scoville, N., Aussel, H., Brusa, M., et al. 2007, ApJS, 172, 1

Tody, D., Dolensky, M., \& Mc Dowell, J., e. a. 2008, Simple Spectra Access Protocol, Version 1.04 (IVOA Recommendation)

Tonry, J. \& Davis, M. 1979, AJ, 84, 1511

Vettolani, G., Zucca, E., Zamorani, G., et al. 1997, A\&A, 325, 954

This 2-column preprint was prepared with the AAS LATEX macros v5.2. 
TABLE 1

EXAMPLES OF RELIABILITY WEIGHTING SCHEME

\begin{tabular}{|c|c|c|c|c|c|}
\hline $\begin{array}{l}\text { Feature } \\
\text { name }\end{array}$ & wavelength & $\begin{array}{l}\text { secondary } \\
\text { feature }\end{array}$ & $\begin{array}{l}\text { weight if } \\
\text { all found }\end{array}$ & $\begin{array}{c}\text { weight if secondary } \\
\text { not found }\end{array}$ & $\begin{array}{l}\text { weight if } \\
\text { not found }\end{array}$ \\
\hline \multicolumn{6}{|c|}{ StarBurst galaxy } \\
\hline$[\mathrm{OII}]$ & 3727.5 & & 10 & & -20 \\
\hline $\mathrm{H} \beta$ & 4861.3 & $\mathrm{H} \gamma$ & 25 & 10 & -20 \\
\hline$[\mathrm{OIII}]_{a}$ & 5006.8 & {$[\mathrm{OIII}]_{b}$} & 30 & 10 & -20 \\
\hline $\mathrm{H} \alpha$ & 6562.8 & SII & 25 & 10 & -20 \\
\hline \multicolumn{6}{|c|}{ Elliptical galaxy } \\
\hline D4000 & 4000 & $\mathrm{CaH} \mathrm{CaK}$ & 50 & 20 & -10 \\
\hline $\mathrm{G}_{\text {band }}$ & 4304.4 & & 10 & & 0 \\
\hline $\mathrm{H} \gamma$ & 4340.4 & & 10 & & 0 \\
\hline MgI & 5175.4 & & 10 & & 0 \\
\hline $\mathrm{Ca}+\mathrm{Fe}$ & 5269.0 & & 10 & & 0 \\
\hline $\mathrm{NaD}$ & 5892.5 & & 10 & & 0 \\
\hline
\end{tabular}

NotE.-The weighting scheme presented is the one adopted for the VVDS and zCosmos bright surveys

TABLE 2

SignificAnCE OF VVDS FLAGS AND CRITERIA FOR EZ FLAGS

\begin{tabular}{cccc}
\hline \hline Flag & $\begin{array}{c}\text { VVDS } \\
\text { confidence }\end{array}$ & EZ lines & rate \\
\hline 4 & $>95 \%$ & $N_{\text {det }} / N_{\text {exp }}>0.5$ & high \\
3 & $90 \%$ & $N_{\text {det }} / N_{\text {exp }}>0.5$ & low \\
2 & $75 \%$ & $N_{\text {det }} / N_{\text {exp }}<0.5$ & high \\
9 & $75 \%$ & $N_{\text {det }}=1$ & high \\
1 & $50 \%$ & $N_{\text {det }} / N_{\text {exp }}<0.5$ & low \\
0 & $0 \%$ & $N_{\text {det }}=0$ & low \\
\hline
\end{tabular}

Note. $-N_{\exp }$ and $N_{\text {det }}$ indicate the number of expected and detected spectral features respectively. See section 6 for a detailed explanation. 
TABLE 3

EZ PERFORMANCES ON SIMULATED DATA

\begin{tabular}{cccc}
\hline \hline EZ flag & SR & Ncorrect & Ntotal \\
\hline \multicolumn{4}{c}{ Galaxies } \\
\hline \multicolumn{4}{c}{10934} \\
any & $92 \%$ & 10092 & 9709 \\
$3-4$ & $95 \%$ & 9307 & 40 \\
2 & $35 \%$ & 14 & 717 \\
9 & $98 \%$ & 708 & 144 \\
1 & $29 \%$ & 43 & 324 \\
0 & $6 \%$ & 20 & 3122 \\
\multicolumn{4}{c}{ Stars } \\
\hline any & $99 \%$ & 8103 & 3321 \\
$3-4$ & $99 \%$ & 4320 & 129 \\
2 & $99 \%$ & 129 \\
1 & $100 \%$ & 3 & 3668 \\
0 & $99 \%$ & 3651 & \\
\hline \multicolumn{4}{c}{ Galaxies and stars } \\
\hline any & $97 \%$ & 18196 & 21955 \\
$3-4$ & $97 \%$ & 13627 & 14030 \\
2 & $91 \%$ & 143 & 170 \\
9 & $98 \%$ & 708 & 717 \\
1 & $42 \%$ & 46 & 147 \\
0 & $93 \%$ & 3671 & 3992 \\
\hline
\end{tabular}

Note. - The success rate (SR) is defined as the percentage of simulated spectra (Ntotal) that are assigned a correct redshift (Ncorrect). These are tabulated as a function of the EZ flag described in the text. 
TABLE 4

EZ PERFORMANCES ON REAL DATA

\begin{tabular}{ccccc}
\hline \hline EZ flag & SR & Ncorrect & Ntotal & SR weighted \\
\hline \multicolumn{5}{c}{ VVDS Deep } \\
\hline any & $68 \%$ & 5833 & 8514 & $76 \%$ \\
$3-4$ & $91 \%$ & 3845 & 4191 & $94 \%$ \\
2 & $51 \%$ & 220 & 427 & $61 \%$ \\
9 & $75 \%$ & 730 & 962 & $82 \%$ \\
1 & $48 \%$ & 372 & 762 & $58 \%$ \\
0 & $30 \%$ & 666 & 2172 & $40 \%$ \\
\hline \multicolumn{5}{c}{ VVDS Wide } \\
\hline any & $70 \%$ & 12242 & 17436 & $78 \%$ \\
$3-4$ & $89 \%$ & 9091 & 10126 & $93 \%$ \\
2 & $62 \%$ & 810 & 1288 & $72 \%$ \\
9 & $51 \%$ & 263 & 509 & $59 \%$ \\
1 & $50 \%$ & 698 & 1381 & $56 \%$ \\
0 & $33 \%$ & 1381 & 4132 & $43 \%$ \\
\hline \multicolumn{5}{c}{ zCosmos Bright } \\
\hline any & $76 \%$ & 6403 & 8404 & $80 \%$ \\
$3-4$ & $97 \%$ & 3735 & 3822 & $98 \%$ \\
2 & $87 \%$ & 445 & 510 & $90 \%$ \\
9 & $57 \%$ & 194 & 336 & $62 \%$ \\
1 & $53 \%$ & 677 & 1260 & $59 \%$ \\
0 & $54 \%$ & 1352 & 2476 & $62 \%$ \\
\hline
\end{tabular}


TABLE 5

EZ AND HUMANLY ASSIGNED FLAGS COMPARISON

\begin{tabular}{cccc}
\hline \hline \multirow{4}{*}{ EZ flag } & \multicolumn{3}{c}{ human flag } \\
\cline { 2 - 4 } & & $2-9$ \\
\hline & \\
\hline \multicolumn{4}{c}{ VVDS Deep } \\
\hline $3-4$ & $84 \%$ & $15 \%$ & $1 \%$ \\
$2-9$ & $42 \%$ & $54 \%$ & $4 \%$ \\
1 & $55 \%$ & $41 \%$ & $4 \%$ \\
0 & $39 \%$ & $52 \%$ & $9 \%$ \\
\hline \multicolumn{4}{c}{ VVDS Wide } \\
\hline $3-4$ & $72 \%$ & $24 \%$ & $3 \%$ \\
$2-9$ & $26 \%$ & $61 \%$ & $12 \%$ \\
1 & $39 \%$ & $52 \%$ & $9 \%$ \\
0 & $24 \%$ & $56 \%$ & $20 \%$ \\
\hline \multicolumn{4}{c}{ zCosmos Bright } \\
\hline $3-4$ & $89 \%$ & $9 \%$ & $2 \%$ \\
$2-9$ & $58 \%$ & $36 \%$ & $6 \%$ \\
1 & $74 \%$ & $20 \%$ & $5 \%$ \\
0 & $40 \%$ & $44 \%$ & $15 \%$ \\
\hline
\end{tabular}

TABLE 6

COMPlETENeSS AND PURITY OF THE SIMUlated GALAXY SAMPle

\begin{tabular}{cccccc}
\hline \hline flag & Ninput & Nmeasured & Ncorrect & Completeness & Purity \\
\hline any & 10934 & 10762 & 10092 & $98.4 \%$ & $93.8 \%$ \\
$3-4$ & 10934 & 9709 & 9307 & $88.8 \%$ & $95.8 \%$ \\
$2 ; 3 ; 4 ; 9$ & 10934 & 10466 & 10029 & $95.7 \%$ & $91.7 \%$ \\
\hline
\end{tabular}

Note. - Completeness is defined as the number of measured redshifts over the total number of input spectra, purity is defined the fraction of correctly recovered redshifts with respect to the measured ones 
TABLE 7

Purity of the ReAl Data SAMple

\begin{tabular}{ccccc}
\hline \hline sample & Ntotal & EZ flag 3-4 & purity & gain \\
\hline VVDS-Deep & 9742 & 3845 & $91 \%$ & $40 \%$ \\
VVDS Wide & 18984 & 10126 & $89 \%$ & $53 \%$ \\
zCosmos Bright & 8404 & 3822 & $97 \%$ & $45 \%$ \\
\hline
\end{tabular}

Noте.-Gain is defined as the percentage of spectra for which human measurement can be spared 


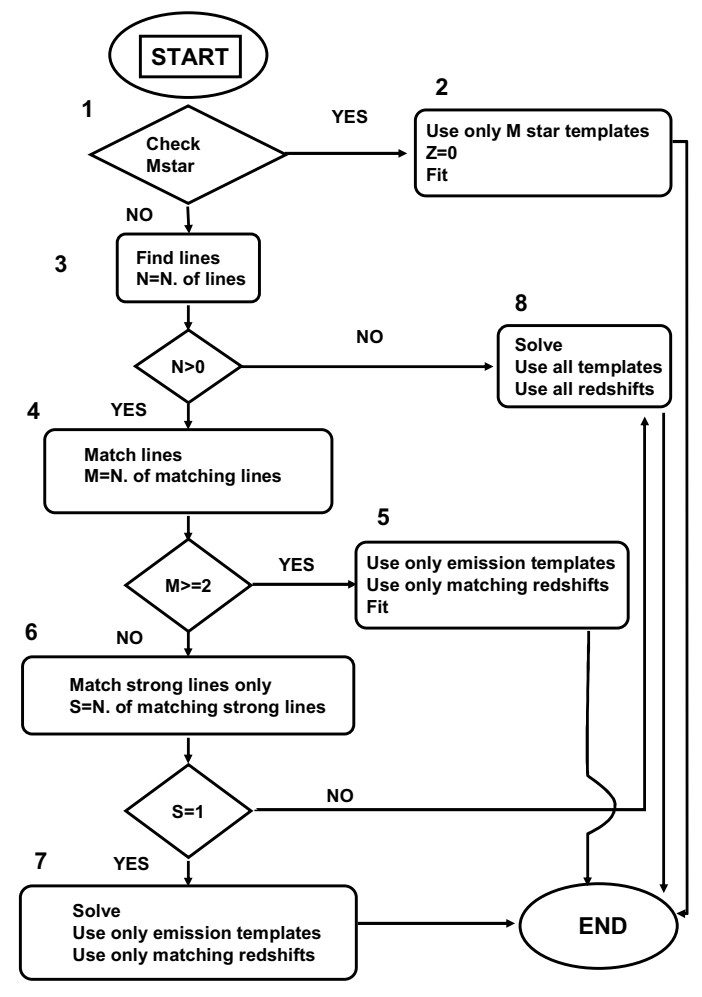

Fig. 2.- Example of EZ decisional tree
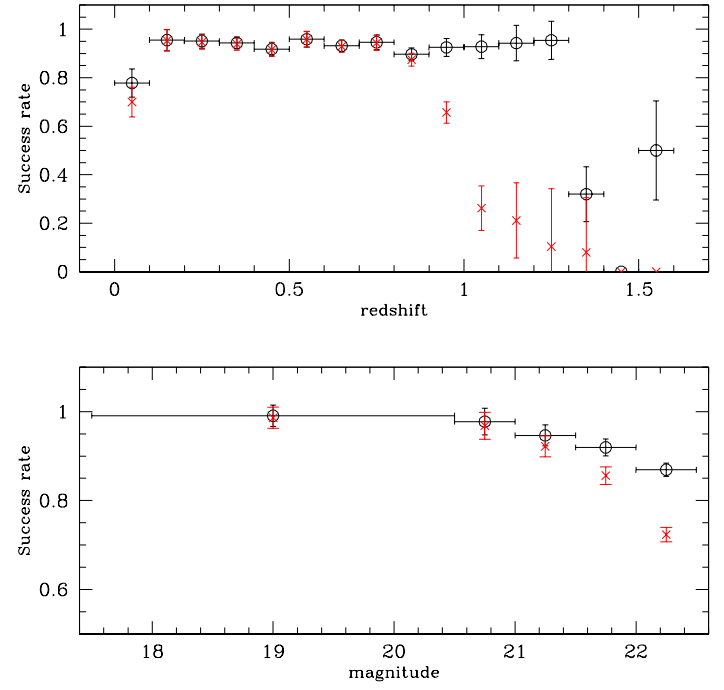

Fig. 3.- EZ success rate as a function of magnitude (lower panel) and of redshift (upper panel), considering flags 2 and above (black open circles) and only flags 3 and 4 (red crosses). Horizontal error bars indicate the bin width, vertical error bars are poissonian errors

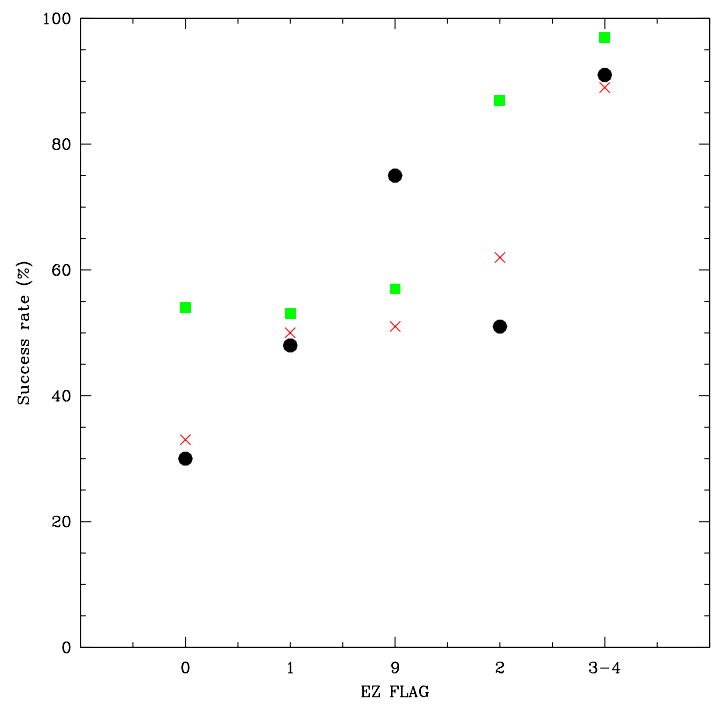

Fig. 4.- EZ success rate as a function of reliability flag. Black dots for VVDS Deep sample, red crosses for VVDS Wide sample, green squares for zCosmos bright sample 


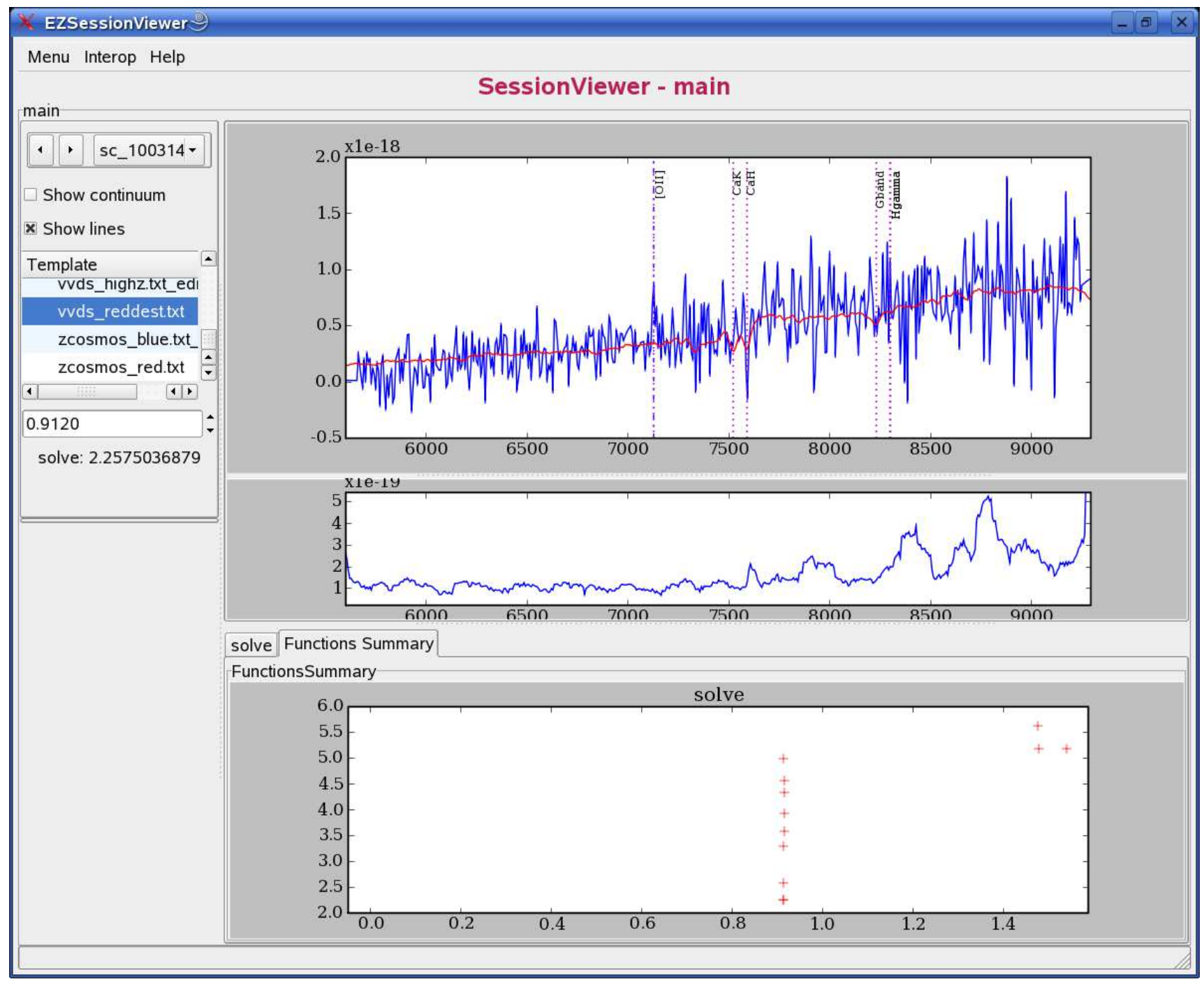

Fig. 1.- EZ Graphical User Interface. In this figure we show a low signal to noise spectrum with the purpose of demonstrating EZ capabilities also on low quality data. Top panel: observed spectrum with best fitting template superimposed (red line). Most important emission or absorption lines are shown as vertical dotted red lines, emission lines found by EZ are marked with vertical blue dashed-dotted line. Middle panel: the noise spectrum. Bottom panel: the best reduced $\chi^{2}$ found for each template as a function of redshift. Left panel: top, pull down menu with the list of currently loaded spectra; middle, the list of available templates, the one currently shown is highlighted; bottom, the redshift of the currently displayed solution (default to the best solution found) and the corresponding reduced $\chi^{2}$ 